\title{
Síndrome de Horner como motivo de consulta en paciente con tumor de mama. Reporte de caso
}

\section{Horner syndrome as a reason for consultation in a patient with breast tumor; case report}

\author{
Verónica C. Martínez-Mayorquín ${ }^{*}$, David Lozano-Elizondo², Rosa E. Moreno-Anda³, Luis M. Pesci-Eguía², \\ Karina L. Miranda-Sánchez ${ }^{1}$ y Irene González-Olhovich² \\ ${ }^{1}$ Instituto Nacional de Neurología y Neurocirugía Manuel Velasco Suárez; ${ }^{2}$ Servicio de Neuroftalmología, Instituto Nacional de Neurología y \\ Neurocirugía Manuel Velasco Suárez; ${ }^{3}$ Segmento Anterior, Instituto Nacional de Rehabilitación Dr. Guillermo Ibarra. Ciudad de México, México
}

\section{Resumen}

El síndrome de Horner consiste en una lesión de la vía pupilar simpática. Descrito con la triada: ptosis, miosis y anhidrosis. Reconocer los signos de este síndrome, sus niveles de lesión y las causas es de suma importancia. Caso: Paciente mujer de 38 años de edad, acude por ptosis del párpado superior del ojo derecho de 2 meses de evolución, hace 4 años ganglio axilar, hace 6 meses aparición de tos; pérdida de peso: $6 \mathrm{~kg}$ en 3 meses. Exploración: ptosis leve, anisocoria mayor en la oscuridad, miosis derecha. Se realiza exploración: tumoración en la mama derecha de $6 \mathrm{~cm}$, adherida a planos profundos. Se envía al centro de cancerología. Este caso toma importancia, ya que actualmente existe una amplia difusión de programas de prevención, sin embargo, aún hay personas que siguen escondiendo sus padecimientos, no debemos olvidar que el globo ocular es punto clave para detectar enfermedades sistémicas.

Palabras clave: Síndrome de Horner. Miosis. Ptosis. Mama.

\begin{abstract}
Horner's syndrome consists of an injury to the sympathetic pupillary pathway. Described with the triad: ptosis, miosis and anhidrosis. Recognizing the signs of this syndrome, their levels of injury and causes are of paramount importance to the clinician. Case: A 38-year-old female patient presented with ptosis of the upper eyelid, right eye, 2 months of evolution, growth of axillary ganglion 4 years ago, also cough of 6 month duration; $6 \mathrm{~kg}$ weight loss in 3 months. Exploration: mild ptosis, greater anisocoria in the dark, right miosis. Sistemic exploration: right breast tumor of 6cm, adhered to deep planes. The patient was referred to an oncological center. This case is important because there is a wide spread of prevention programs, however, there are still people who continue to hide their diseases, we must not forget that the eyeball is a key point to detect systemic diseases.
\end{abstract}

Key words: Horner Syndrome. Miosis. Ptosis. Breast.

Correspondencia:

*Verónica Cristina Martínez-Mayorquín

Juan Manuel, 1500

Col. Ladrón de Guevara

Fecha de recepción: 12-06-2017

Fecha de aceptación: 22-11-2017

Disponible en internet: 27-07-2018

C.P. 44600 , Guadalajara, Jal., México

DOI: 10.24875/RMO.M18000026

Rev Mex Oftalmol. 2019;93(5):258-261

E-mail: draveronicacristina@gmail.com www.rmo.com.mx

0187-4519/○ 2017 Sociedad Mexicana de Oftalmología. Publicado por Permanyer México. Este es un artículo Open Access bajo la licencia CC BY-NC-ND (http://creativecommons.org/licenses/by-nc-nd/4.0/). 


\section{Introducción}

El síndrome de Horner fue descrito por primera vez en 1869 por el oftalmólogo suizo Johann Friedrich Horner. De forma clásica está conformado por una lesión simpática y con la triada de: ptosis, miosis y anhidrosis, dependiendo del nivel donde se encuentre la lesión. No siempre la triada se cumple, podemos encontrar hasta un $10 \%$ de los pacientes sin ptosis. Las causas son múltiples, como disección carotidea, tumoraciones que comprometen el ápice pulmonar y lesiones hipotalámicas, entre otras. Es importante mencionar que puede ser una manifestación o signo de enfermedad metastásica. Reconocer los signos de este síndrome, sus niveles de lesión y sus implicaciones es de suma importancia para el clínico.

Existen pruebas farmacológicas destinadas a su reconocimiento, cuyos objetivos son: reconocer que existe anisocoria (la cual es significativa clínicamente de $1 \mathrm{~mm}$ o más de diferencia), identificar si se trata de un problema autonómico o intrínseco pupilar y localizar una lesión a nivel posganglionar. Sin embargo, existen signos muy sugestivos y localizadores que nos permiten realizar el diagnóstico. Además, es importante interrogar por aquellos síntomas acompañantes para lograr localizar el nivel de la lesión.

Los síntomas pupilares suelen ser sutiles, ya que anatómicamente la pupila en su control nervioso (simpático y parasimpático) tiene una proporción de fibras parasimpáticas y simpáticas 30:1. Por lo que la anisocoria no es muy evidente en condiciones de luz normal. Podemos encontrar frecuentemente síndrome de Horner asociado a parálisis del VI par craneal ipsilateral «aislado». Es indispensable explorar a los pacientes detenidamente y con diferentes condiciones de luz, para poder clasificar los hallazgos que encontremos y realizar un diagnóstico topográfico más certero.

\section{Presentación de caso}

Mujer de 38 años de edad, enviada al Servicio de Neuroftalmología del Instituto Nacional de Neurología y Neurocirugía Manuel Velasco Suárez por presentar ptosis del párpado superior del ojo derecho de 2 meses de evolución.

\section{Principio y evolución del padecimiento actual}

Refiere hace 4 años ganglio axilar que fue aumentando de tamaño, y hace 6 meses aparición de tos no productiva y crecimiento de ganglios cervicales (6 aproximadamente, pétreos, no móviles e irregulares); pérdida de peso: $6 \mathrm{~kg}$ en 3 meses.

\section{Hallazgos}

Ptosis leve en el ojo derecho (2 $\mathrm{mm}$ comparado con el contralateral), anisocoria mayor en condiciones de poca iluminación con miosis derecha, apertura del párpado del ojo derecho de $9 \mathrm{~mm}$, distancia margen reflejo 1 (DRM 1) de $2 \mathrm{~mm}$; DMR 2 de $7 \mathrm{~mm}$. Apertura palpebral del ojo izquierdo de $12 \mathrm{~mm}$, DMR 1 de $4 \mathrm{~mm}$, DMR 2 de $8 \mathrm{~mm}$ (Fig. 1). Diámetro pupilar derecho de $3 \mathrm{~mm}$, diámetro pupilar izquierdo de $5 \mathrm{~mm}$, con respuesta a la luz normal bilateral, reflejo consensual presente (Fig. 2). Se le solicita a la paciente descubrir su torso, y se encuentra tumoración en la mama derecha que altera su estructura y por lo que pierde completamente su anatomía, además se palpa dicha tumoración adherida a planos profundos y no se delimita su extensión, la piel se aprecia con características de naranja (Fig. 3). Se solicita tomografía axial computarizada de tórax contrastada y se envía al centro de cancerología para un manejo adecuado. Sin embargo, la paciente no acude a dicho centro y no vuelve a revisión.

\section{Discusión}

En el síndrome de Horner, los signos clínicos pueden ser sutiles y no es común que los pacientes presenten la tríada clásica. Si asociamos los signos y síntomas clínicos, pueden ayudar a localizar la lesión y dirigir el abordaje diagnóstico. En cuanto a las pruebas farmacológicas, sin restar importancia a su utilidad, en nuestro medio son de uso limitado, ya que en ocasiones no se encuentran al alcance y se han encontrado falsos positivos y negativos ${ }^{1}$.

Si bien es cierto que las pruebas medicamentosas, como la cocaína, la hidroxianfetamina y la apraclonidina, nos apoyan importantemente en el diagnóstico, el nivel de la lesión puede ser determinado por los signos clínicos clásicos y los datos acompañantes. Es importante recordar que en esta patología el reflejo pupilar fotomotor se encuentra sin alteraciones ${ }^{1,2}$. No existen pruebas farmacológicas infalibles para determinar el nivel de la lesión.

Se han reportado casos con otras etiologías, como nódulos tiroideos, que en ocasiones remiten después del tratamiento quirúrgico de la enfermedad de base ${ }^{2}$. En relación con el caso clínico que se presenta, se han publicado reportes de metástasis de cáncer de mama 


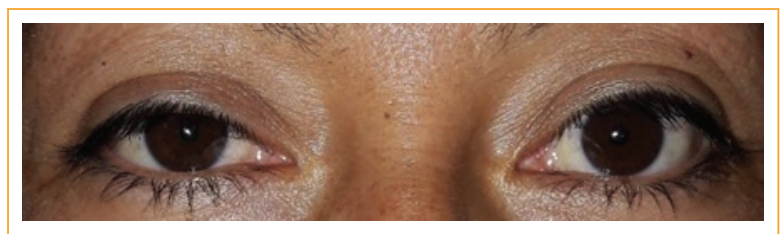

Figura 1. Exploración en condiciones de luz con apertura de párpado. Ojo derecho: $9 \mathrm{~mm}$, distancia margen reflejo 1 (DMR 1): $2 \mathrm{~mm}$. DMR 2: $7 \mathrm{~mm}$. Ojo izquierdo apertura: $12 \mathrm{~mm}$. DMR 1: $4 \mathrm{~mm}$. DMR 2: $8 \mathrm{~mm}$. Anisocoria.

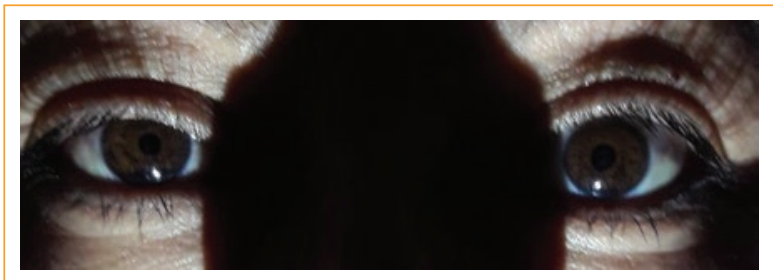

Figura 2. Anisocoria mayor en la oscuridad. Diámetro pupilar derecho: $3 \mathrm{~mm}$. Diámetro pupilar izquierdo: $5 \mathrm{~mm}$. Respuesta a la luz normal.

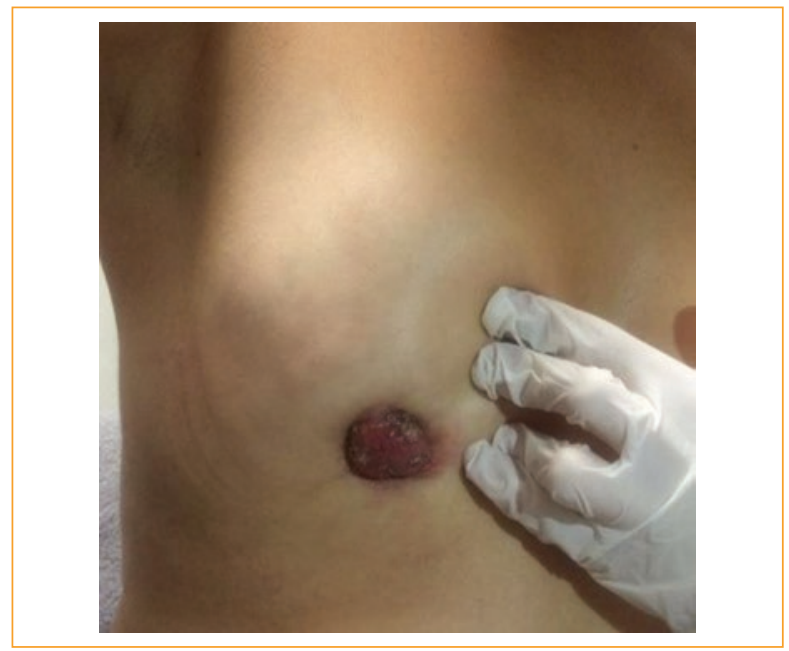

Figura 3. Tumoración de mama derecha.

que se presentan como síndrome de Horner ${ }^{3}$. A diferencia de nuestro caso, la paciente no contaba con diagnóstico de malignidad previamente.

Entre otras causas de síndrome de Horner encontramos la disección de la arteria carótida interna, como resultado de un accidente y/o trauma en pacientes menores de 45 años de edad, causante también del 10 a $20 \%$ de los casos de accidente cerebrovascular isquémico. El diagnóstico debe sospecharse con la combinación del síndrome de Horner, dolor de cabeza 0 de cuello y signos de isquemia retiniana 0 cerebral ${ }^{4}$.

Otras causas de lesión de la vía pupilar simpática menos frecuentes son enfermedades inflamatorias del sistema nervioso central, como neuromielitis óptica o enfermedad de Devic. Se reporta el caso de una mujer de 58 años que presentó síndrome de Horner con hemianestesia contralateral, confirmado con $0.5 \%$ de apraclonidina y títulos positivos anticuerpos antiacuaporina $4^{5}$.

El síndrome de Horner puede clasificarse en tres grupos según la localización de la lesión. Central, el cual puede ocurrir en lesiones de hipotálamo, tallo cerebral y la médula espinal que afectan a la vía simpática. Las entidades más frecuentemente vistas son síndrome de Wallenberg y siringomielia, entre otras. Los hallazgos neurológicos concomitantes podrían sugerir este tipo de lesiones. El tipo preganglionar puede ser resultado de las lesiones radiculares en la médula espinal, plexo braquial, trauma, lesiones del ápice pulmonar o tumores mediastínicos. La lesión posganglionar está relacionada con enfermedades de la arteria carótida interna, base del cráneo, enfermedades del seno cavernoso, fisura orbitaria superior y enfermedades del ápice orbitario, entre otras. El enoftalmos es aparente por causa de la ptosis inversa, ya que existe pérdida de tono simpático del análogo del músculo de Müller inferior, o músculo tarsal inferior 6 .

En el caso de lesión de neurona de tercer orden, las fibras simpáticas responsables de la función sudomotora de la porción inferior del rostro se separan de la vía oculosimpática antes del ganglio cervical superior, por lo tanto, la anhidrosis, raramente se observará en esta distribución en lesiones posganglionares, por lo que se debe explorar la sudoración en el tercio superior de la cara ${ }^{7}$. Podemos buscar de forma sencilla este dato, aplicando talco al paciente en el rostro y pidiéndole que realice actividad física para observar si algún lado de la cara no presenta sudoración, o de forma más conocida utilizando yodo.

La cocaína es un simpaticomimético que bloquea la recaptación de norepinefrina. Mientras que las pupilas normales se dilatan, en una lesión del simpático no se dilatan, ya que la vía de las tres neuronas ha sido interrumpida. La apraclonidina (agonista alfa-2-adrenérgico) tópica también es una opción en pruebas medicamentosas. Se ha demostrado que este fármaco revierte la anisocoria al dilatar la pupila patológica y no alterar la pupila normal. La hidroxianfetamina tópica al 1\% también se puede utilizar para confirmar lesión de la tercera neurona o posganglionar. La hidroxianfetamina estimula 
la liberación de norepinefrina en botón presináptico. En las lesiones de la primera o segunda neurona el botón presináptico está intacto, por lo que la pupila se dilata, en las lesiones de la tercera neurona no ${ }^{8-10}$.

Las pupilas deben ser valoradas minuciosamente siempre en diferentes condiciones de luz y con luz adecuada, buscando si existe anisocoria o alteración en los reflejos pupilares. Si detectamos respuestas anormales, valorar si es unilateral o bilateral y si está acompañada de otros síntomas e interrogar adecuadamente el paciente. Si seguimos estos pasos, es menos probable que dejemos pasar signos importantes para un diagnóstico crucial.

\section{Conclusión}

En el presente artículo se revisaron las características generales y las diferentes causas del síndrome de Horner. En el caso de nuestra paciente, es importante notar que el motivo de consulta fue "ptosis leve", sin mención alguna de una malignidad u otra patología de base. El interrogatorio por aparatos y sistemas fue difícil, ya que la paciente tenía una conducta retraída y negaba otros síntomas y signos. Sin embargo, al seguir la cadena ganglionar involucrada, se detectó afectación de los ganglios axilares. Como los ganglios axilares en una mujer joven son sugestivos de una malignidad de origen mamario, se realizó una exploración de tórax y se encontró una lesión altamente sugestiva de cáncer de mama.

Este caso toma relevancia, ya que actualmente se piensa que en nuestra sociedad hay información vasta y programas de prevención suficientes, sin embargo, hoy en día existen personas que siguen escondiendo sus padecimientos hasta que llegan a estadios avanzados, enfermedad metastásica o muerte. Es por ello que al encontrar un hallazgo debemos de indagar, investigar, observar y realizar una historia clínica exhaustiva.

A pesar de explicarle a la paciente la urgencia de acudir al centro oncológico, solicitarle estudios de gabinete y darle una nueva cita a control en nuestro servicio, la paciente no volvió y perdió su seguimiento.

Desde el punto de vista neuroftalmológico no debemos olvidar que tras cada globo ocular existe un paciente con todo un sistema que funciona en conjunto y que muchas patologías pueden tener su primera manifestación en el sistema visual.

Es nuestra obligación realizar una exploración completa y una adecuada historia clínica para llegar a la etiología. Saber y conocer la función, sensibilidad y especificidad de las pruebas farmacológicas para la detección de síndrome de Horner es indispensable, pero no supera el aporte de la semiología bien llevada a cabo.

\section{Responsabilidades éticas}

Protección de personas y animales. Los autores declaran que para esta investigación no se han realizado experimentos en seres humanos ni en animales.

Confidencialidad de los datos. Los autores declaran que han seguido los protocolos de su centro de trabajo sobre la publicación de datos de pacientes.

Derecho a la privacidad y consentimiento informado. Los autores han obtenido el consentimiento informado de los pacientes y/o sujetos referidos en el artículo. Este documento obra en poder del autor de correspondencia.

\section{Bibliografía}

1. Davagnanam I, Fraser CL, Miszkiel K, Daniel CS, Plant GT. Adult Horner's syndrome: a combined clinical, pharmacological, and imaging algorithm. Eye. 2013;27:291-8

2. Coskun M, Aydogan A, Gokce C, Ilhan O, Ozkan OV, Gokce H, Oksuz H. Irreversible Horner's syndrome diagnosed by aproclonidine test due to benign thyroid nodule. Pak J Med Sci. 2013;29(1):224-6.

3. Zhou D, Ibrahim M, Malach D, Tomsak RL. Unusual Cause of Horner Syndrome Years After In Situ Ductal Carcinoma. Neuroophthalmology. 2016; 40(3):130-2

4. Alonso Formento JE, Fernández Reyes JL, Envid Lázaro BM, Fernández Letamendi T, Yeste Martín R, Jódar Morente FJ. Horner's Syndrome 17 due to a Spontaneous Internal Carotid Artery Dissection after Deep Sea Scuba Diving. Case Rep Neurol Med.2016;2016:5162869.

5. Lovera L, Jay WM, Biller J. Horner Syndrome in a Case of Neuromyelitis Optica. Neuroophthalmology. 2014;38(2):78-81.

6. Jacob S, Patil SI. Heralding Extramedullary Blast Crisis: Horner's Syndrome with Brachial Plexopathy in a Patient with Chronic Myelogenous Leukemia. Case Reports in Medicine. 2016;2016:3015947.

7. Nasser BA, Mesned A, Moazamy YE, Kabbani MS. Horner's syndrome after paediatric cardiac surgery: case report and review of the literatura. Cardiology Young. 2015;25:569-72.

8. Mohyudin MN, Arshad FA, Anand N. Horner's síndrome as a presenting sign of metastatic testicular malignancy. JRSM Short Rep. 2011;2:23.

9. Manasa Sriraam L, Sundaram R, Ramalingam R, Ramalingam KK. Minor's Test: Objective Demonstration of Horner's Syndrome. Indian J Otolaryngol Head Neck Surg. 2015;67(2):190-2.

10. Rodríguez-Sánchez E, Vadillo JM, Herrera-Calo P, Marenco de la Fuente ML. Síndrome de Horner tras analgesia epidural para el parto. Informe de 3 casos. Rev Colomb Anestesiol. 2016;44(2):170-3. 